\title{
On Rao's Theorems and the Lazarsfeld-Rao Property
}

\author{
Robin Hartshorne
}

\begin{abstract}
Let $X$ be an integral projective scheme satisfying the condition $S_{3}$ of Serre and $H^{1}\left(\mathcal{O}_{X}(n)\right)=0$ for all $n \in \mathbb{Z}$. We generalize Rao's theorem by showing that biliaison equivalence classes of codimension two subschemes without embedded components are in one-to-one correspondence with pseudo-isomorphism classes of coherent sheaves on $X$ satisfying certain depth conditions.

We give a new proof and generalization of Strano's strengthening of the LazarsfeldRao property, showing that if a codimension two subscheme is not minimal in its biliaison class, then it admits a strictly descending elementary biliaison.

For a three-dimensional arithmetically Gorenstein scheme $X$, we show that biliaison equivalence classes of curves are in one-to-one correspondence with triples $(M, P, \alpha)$, up to shift, where $M$ is the Rao module, $P$ is a maximal Cohen-Macaulay module on the homogeneous coordinate ring of $X$, and $\alpha: P^{\vee} \rightarrow M^{*} \rightarrow 0$ is a surjective map of the duals.
\end{abstract}

\section{Introduction}

The Lazarsfeld-Rao property was initially formulated for curves in $\mathbb{P}^{3}$. It says that each even liaison class of curves has minimal elements, which form an irreducible family. Any curve in the even liaison class that is not minimal can be obtained from a minimal curve by a finite sequence of ascending basic double links, followed by a deformation. This result has been generalized in several directions, and most recently Strano [15] has shown that if one uses elementary biliaisons, the deformation at the last step is not necessary. Note that a basic double link is essentially a union of the smaller scheme with a complete intersection, while an elementary biliaison has linear equivalence built into the definition. So another way of phrasing Strano's result is that the only deformations needed in the Lazarsfeld-Rao theorem are linear equivalences on suitable hypersurfaces.

Our purpose in this paper is to give a uniform treatment of the theorems of Rao and the Lazarsfeld-Rao property, in a fairly general context, and from a slightly different point of view than the traditional one. In particular, we will use biliaisons only, and we deal with codimension 2 subschemes that are equidimensional and without embedded components. 
A good summary of the literature can be found in Chapter 6 of Migliore's book [10]. Rao first gave theorems relating biliaison equivalence classes of codimension 2 subschemes to stable equivalence classes of certain sheaves on $\mathbb{P}^{n}$ 13, 14. The Lazarsfeld-Rao property was first proved in a special case in [8]. Then it was proved independently for curves in $\mathbb{P}^{3}$ in [9] and for codimension 2 locally Cohen-Macaulay schemes in [1. The ambient scheme was generalized from $\mathbb{P}^{n}$ to an arithmetically Gorenstein scheme in [14] and 2]. The condition that the subschemes be locally Cohen-Macaulay was dropped in [11] and [12. The necessity of the deformation at the last step of the LR property was eliminated by Strano [15].

In this paper we retain all these generalizations. Using ideas from the papers mentioned above, and some technical results from the papers [5], [6], we can now prove the LazarsfeldRao property for biliaison equivalence classes of codimension 2 subschemes without embedded components on an integral projective scheme $X$ satisfying condition $S_{3}$ of Serre and $H_{*}^{1}\left(\mathcal{O}_{X}\right)=0(2.4)$. We also give a version of the theorem of Rao for a three-dimensional arithmetically Gorenstein scheme (3.2). We have made an effort in this paper to present each result with the minimal number of hypotheses, so as to bring out more clearly which results depend on which assumptions.

\section{Pseudo-isomorphism}

Here we borrow some ideas from [5. Section 2] and adapt them to our situation.

Hypotheses 1.1. Let $X$ be a projective scheme over an algebraically closed field $k$, equidimensional of dimension $N \geq 2$. We assume $X$ satisfies the condition $S_{2}$ of Serre. Let $\mathcal{O}_{X}(1)$ be an ample invertible sheaf. For any coherent sheaf $\mathcal{F}$ we denote $\oplus_{n \in \mathbb{Z}} H^{i}(X, \mathcal{F}(n))$ by $H_{*}^{i}(\mathcal{F})$. We assume that $H_{*}^{1}\left(\mathcal{O}_{X}\right)=0$.

Definition 1.2. A coherent sheaf $\mathcal{L}$ on $X$ is dissocié if $\mathcal{L} \cong \oplus \mathcal{O}_{X}\left(n_{i}\right)$ for some $n_{i} \in \mathbb{Z}$.

Definition 1.3. An elementary pseudo-isomorphism is a surjective map $\mathcal{E} \rightarrow \mathcal{E}^{\prime} \rightarrow 0$ of coherent sheaves whose kernel is dissocié. The equivalence relation generated by the elementary pseudo-isomorphisms (and their inverses) is called pseudo-isomorphism (psi for short).

Lemma 1.4. A composition of elementary psi is an elementary psi.

Proof. Suppose given

$$
\begin{aligned}
& 0 \rightarrow \mathcal{L} \rightarrow \mathcal{E} \rightarrow \mathcal{E}^{\prime} \rightarrow 0 \\
& 0 \rightarrow \mathcal{L}^{\prime} \rightarrow \mathcal{E}^{\prime} \rightarrow \mathcal{E}^{\prime \prime} \rightarrow 0
\end{aligned}
$$


with $\mathcal{L}$ and $\mathcal{L}^{\prime}$ dissocié. The kernel of the composed map $\mathcal{E} \rightarrow \mathcal{E}^{\prime \prime}$ is an extension of $\mathcal{L}^{\prime}$ by $\mathcal{L}$. Because of the hypothesis $(1.1)$ that $H_{*}^{1}\left(\mathcal{O}_{X}\right)=0$, this extension splits, so the kernel is $\mathcal{L} \oplus \mathcal{L}^{\prime}$, which is dissocié.

Lemma 1.5. If $\mathcal{E}_{1}$ and $\mathcal{E}_{2}$ are psi-equivalent, then there exists a coherent sheaf $\mathcal{F}$ and elementary psi's $\mathcal{F} \rightarrow \mathcal{E}_{1}$ and $\mathcal{F} \rightarrow \mathcal{E}_{2}$.

Proof. Any psi is a finite composition of elementary psi's and their inverses. In view of (1.4), and using induction on the length of a chain, it is sufficient to show that if $\mathcal{E}_{1} \rightarrow \mathcal{E}^{\prime}$ and $\mathcal{E}_{2} \rightarrow \mathcal{E}^{\prime}$ are elementary psi's, then there exists elementary psi's $\mathcal{F} \rightarrow \mathcal{E}_{1}$ and $\mathcal{F} \rightarrow \mathcal{E}_{2}$ making a commutative diagram. So let

$$
0 \rightarrow \mathcal{L}_{1} \rightarrow \mathcal{E}_{1} \rightarrow \mathcal{E}^{\prime} \rightarrow 0
$$

and

$$
0 \rightarrow \mathcal{L}_{2} \rightarrow \mathcal{E}_{2} \rightarrow \mathcal{E}^{\prime} \rightarrow 0
$$

Take $\mathcal{F}$ to be the fibered sum $\operatorname{ker}\left(\mathcal{E}_{1} \oplus \mathcal{E}_{2} \rightarrow \mathcal{E}^{\prime}\right)$. Then the kernel of the natural map $\mathcal{F} \rightarrow \mathcal{E}_{i}$ is just $\mathcal{L}_{3-i}$ for $i=1,2$.

Definition 1.6. We say a coherent sheaf $\mathcal{E}$ on $X$ satisfies the condition $T$ if the following hold

1) $\mathcal{E}$ is locally free of constant rank in codimension $\leq 1$.

2) $\mathcal{E}$ has depth $\geq 1$ in codimension 2 .

3) $\mathcal{E}$ has depth $\geq 2$ in codimension $\geq 3$.

4) There is a closed subset $Z \subseteq X$ of codimension $\geq 2$ such that $\mathcal{E}$ is locally free on $X-Z$ and $\left.\operatorname{det} \mathcal{E}\right|_{X-Z} \cong \mathcal{O}_{X-Z}(\ell)$ for some $\ell \in \mathbb{Z}$. In this case, we say $\mathcal{E}$ is orientable.

Example 1.7. a) Let $V$ be a closed subscheme of $X$ of codimension 2, equidimensional, and having no embedded components. Then the ideal sheaf $\mathcal{I}_{V}$ of $V$ satisfies the condition $T$. To each such closed subscheme $V$ then, we will associate the psi-equivalence class of coherent sheaves satisfying $T$ containing the ideal sheaf $\mathcal{I}_{V}$.

b) Conversely, if $\mathcal{E}$ is a rank 1 coherent sheaf satisfying $T$, then $\mathcal{E}$ is isomorphic to $\mathcal{I}_{V}(n)$ for some subscheme $V$ of codimension 2 without embedded points, and some $n \in Z$. Indeed, let $Z$ be a closed set of codimension $\geq 2$ such that $\mathcal{E}$ is locally free on $X-Z$. Then since $\mathcal{E}$ is orientable, $\mathcal{E} \cong \mathcal{O}_{X}(n)$ on $X-Z$ for some $n$. Let $j: X-Z \rightarrow X$ be the inclusion. Then there is a natural map $\alpha: \mathcal{E} \rightarrow j_{*} j^{*} \mathcal{E}$. Since $X$ satisfies $S_{2}, j_{*} j^{*} \mathcal{E} \cong \mathcal{O}_{X}(n)$. Furthermore, the map $\alpha$ is injective because of the depth conditions on $\mathcal{E}$. Hence $\mathcal{E} \cong \mathcal{I}_{V}(n)$ for some 
closed subscheme $V$ of codimension $\geq 2$. It may happen that $\mathcal{E} \cong \mathcal{O}(n)$, in which case $V$ is empty. If $V$ is nonempty, the depth conditions on $\mathcal{E}$ now imply that $V$ is equidimensional of codimension 2, and has no embedded components.

c) If $V$ is a closed subscheme as in a) and b) above, and if $Y$ is a hypersurface of $X$, linearly equivalent to $n H$, containing $V$, where $H$ is the divisor class corresponding to $\mathcal{O}_{X}(1)$, then the exact sequence $0 \rightarrow \mathcal{I}_{Y} \rightarrow \mathcal{I}_{V} \rightarrow \mathcal{I}_{V, Y} \rightarrow 0$ shows that $\mathcal{I}_{V} \rightarrow \mathcal{I}_{V, Y}$ is a psi, since $\mathcal{I}_{Y} \cong \mathcal{O}_{X}(-n)$. Note, however, that $\mathcal{I}_{V, Y}$ does not satisfy $T$, because it is a torsion sheaf with support in codimension 1 along $Y$.

Lemma 1.8. a) If $\mathcal{E} \rightarrow \mathcal{E}^{\prime}$ is an elementary psi, and $\mathcal{E}^{\prime}$ satisfies $T$, then so does $\mathcal{E}$.

b) if $X$ satisfies condition $S_{3}$ of Serre, and if $\mathcal{E}$ is a sheaf satisfying $T$ of rank $\geq 2$, then there exists an elementary psi $\mathcal{E} \rightarrow \mathcal{E}^{\prime}$ to a sheaf $\mathcal{E}^{\prime}$ of rank one less, also satisfying $T$.

Proof. Part a) is obvious. To prove b), let $\mathcal{E}(n)$ be a twist of $\mathcal{E}$ that is generated by global sections. I claim that if $s$ is a sufficiently general section in $H^{0}(\mathcal{E}(n))$, then the quotient $\mathcal{E}^{\prime}=\mathcal{E} / \mathcal{O}(-n)$ defined by $s$ will be locally free in codimension 1 . At a point $x \in X$, the condition for $\mathcal{E}^{\prime}$ to be locally free is that $s(x) \in \mathcal{E}(n) \otimes k(x)$ be non-zero. If $\mathcal{E}$ is locally free of $\operatorname{rank} r \geq 2$, since $\mathcal{E}(n)$ is generated by global sections, the bad set of sections $s \in V=H^{0}(\mathcal{E}(n))$ will have codimension $r$. It follows that for a general $s \in V$, the bad set of $x \in X$ for which $s(x)=0$ will have codimension $\geq r \geq 2$. Hence $\mathcal{E}^{\prime}$ is locally free in codimension 1 . The rest of condition $T$ for $\mathcal{E}^{\prime}$ follows from the $S_{3}$ condition on $X$.

Definition 1.9 [5, 2.6]. A coherent sheaf $\mathcal{E} X$ is extraverti if $H_{*}^{1}\left(\mathcal{E}^{\vee}\right)=0$ and $\mathcal{E} x t^{1}\left(\mathcal{E}, \mathcal{O}_{X}\right)=$ 0 .

Lemma 1.10. a) If $\mathcal{E} \rightarrow \mathcal{E}^{\prime}$ is an elementary psi, and $\mathcal{E}^{\prime}$ is extraverti, so is $\mathcal{E}$.

b) If $\mathcal{E}_{1}$ and $\mathcal{E}_{2}$ are extraverti sheaves that are psi equivalent, then they are stably equivalent, namely, there exist dissocie sheaves $\mathcal{L}$ and $\mathcal{M}$ such that $\mathcal{E}_{1} \oplus \mathcal{L} \cong \mathcal{E}_{2} \oplus \mathcal{M}$.

Proof. a) If $0 \rightarrow \mathcal{L} \rightarrow \mathcal{E} \rightarrow \mathcal{E}^{\prime} \rightarrow 0$ with $\mathcal{L}$ dissocié, then we have

$$
0 \rightarrow \mathcal{E}^{\prime \vee} \rightarrow \mathcal{E}^{\vee} \rightarrow \mathcal{L}^{\vee} \rightarrow \mathcal{E} x t^{1}\left(\mathcal{E}^{\prime}, \mathcal{O}_{X}\right) \rightarrow \mathcal{E} x t^{1}\left(\mathcal{E}, \mathcal{O}_{X}\right) \rightarrow 0 .
$$

Assuming that $\mathcal{E} x t^{1}\left(\mathcal{E}^{\prime}, \mathcal{O}_{X}\right)=0$, we get $\mathcal{E} x t^{1}\left(\mathcal{E}, \mathcal{O}_{X}\right)=0$ and an exact sequence $0 \rightarrow \mathcal{E}^{\prime \vee} \rightarrow$ $\mathcal{E}^{\vee} \rightarrow \mathcal{L}^{\vee} \rightarrow 0$. Now the exact sequence of cohomology shows $H_{*}^{1}\left(\mathcal{E}^{\vee}\right)=0$.

b) In view of part a) and (1.5), it is sufficient to show that if $0 \rightarrow \mathcal{L} \rightarrow \mathcal{E} \rightarrow \mathcal{E}^{\prime} \rightarrow 0$ with $\mathcal{E}^{\prime}$ extraverti, then the sequence splits, so $\mathcal{E} \cong \mathcal{E}^{\prime} \oplus \mathcal{L}$. For this we use the low terms of the spectral sequence of Ext:

$$
0 \rightarrow H_{*}^{1}\left(\mathcal{E}^{\prime \vee}\right) \rightarrow \operatorname{Ext}_{*}^{1}\left(\mathcal{E}^{\prime}, \mathcal{O}_{X}\right) \rightarrow H_{*}^{0}\left(\mathcal{E} x t^{1}\left(\mathcal{E}^{\prime}, \mathcal{O}_{X}\right)\right) \rightarrow H_{*}^{2}\left(\mathcal{E}^{\prime \vee}\right) \rightarrow \ldots
$$

The first and third terms being zero, so is the second, so the sequence splits. 
Proposition 1.11. Every psi equivalence class of sheaves satisfying $T$ contains an extraverti sheaf satisfying $T$.

Proof. Let $\mathcal{E}$ be a coherent sheaf satisfying $T$. Let

$$
0 \rightarrow \mathcal{G} \rightarrow \mathcal{M} \rightarrow \mathcal{E} \rightarrow 0
$$

be a resolution with $\mathcal{M}$ dissocié. From the hypothesis $H_{*}^{1}\left(\mathcal{O}_{X}\right)=0$ we see that $\operatorname{Ext}_{*}^{1}\left(\mathcal{E}, \mathcal{O}_{X}\right)$ is a quotient of $H_{*}^{0}\left(\mathcal{G}^{\vee}\right)$, and hence is a finitely generated graded $S=H_{*}^{0}\left(\mathcal{O}_{X}\right)$-module.

Take a set of generators $\xi_{i} \in \operatorname{Ext}^{1}\left(\mathcal{E}, \mathcal{O}_{X}\left(-a_{i}\right)\right)$ of this module and let

$$
0 \rightarrow \mathcal{L} \rightarrow \mathcal{F} \rightarrow \mathcal{E} \rightarrow 0
$$

be the corresponding extension, with $\mathcal{L}=\oplus \mathcal{O}_{X}\left(-a_{i}\right)$. Then $\mathcal{F}$ is psi-equivalent to $\mathcal{E}$ by construction, and satisfies $T$ by (1.8). We will show that $\mathcal{F}$ is extraverti.

First we apply the functor $\operatorname{Hom}\left(\cdot, \mathcal{O}_{X}(*)\right)$. This gives

$$
0 \rightarrow H_{*}^{0}\left(\mathcal{E}^{\vee}\right) \rightarrow H_{*}^{0}\left(\mathcal{F}^{\vee}\right) \rightarrow H_{*}^{0}\left(\mathcal{L}^{\vee}\right) \stackrel{\alpha}{\rightarrow} \operatorname{Ext}_{*}^{1}\left(\mathcal{E}, \mathcal{O}_{X}\right) \rightarrow \operatorname{Ext}_{*}^{1}\left(\mathcal{F}, \mathcal{O}_{X}\right) \rightarrow 0 .
$$

Furthermore, the map $\alpha$ sends the generators of $H_{*}^{0}\left(\mathcal{L}^{\vee}\right)$ to the chosen generators $\xi_{i}$ of $\operatorname{Ext}_{*}^{1}\left(\mathcal{E}, \mathcal{O}_{X}\right)$. Hence $\operatorname{Ext}_{*}^{1}\left(\mathcal{F}, \mathcal{O}_{X}\right)=0$, and we conclude from the spectral sequence mentioned in the proof of (1.10) above that $H_{*}^{1}\left(\mathcal{F}^{\vee}\right)=0$.

From that same spectral sequence, and noting that $H_{*}^{2}\left(\mathcal{E}^{\vee}\right)$ is zero in large enough degrees, by Serre vanishing, we see that the map

$$
\operatorname{Ext}_{*}^{1}\left(\mathcal{E}, \mathcal{O}_{X}\right) \rightarrow H_{*}^{0}\left(\mathcal{E} x t^{1}\left(\mathcal{E}, \mathcal{O}_{X}\right)\right)
$$

is surjective in large enough degrees. Hence the images of the generators $\xi_{i}$ also generate the sheaf $\mathcal{E} x t^{1}\left(\mathcal{E}, \mathcal{O}_{X}\right)$. Then from applying $\mathcal{H o m}\left(\cdot, \mathcal{O}_{X}\right)$ to the sequence defining $\mathcal{F}$ above, we find also $\mathcal{E} x t^{1}\left(\mathcal{F}, \mathcal{O}_{X}\right)=0$, and $\mathcal{F}$ is extraverti.

Combining the results of this section, we have the following relationship between codimension two subvarieties and coherent sheaves on $X$.

Proposition 1.12. Let $X$ be an equidimensional projective scheme of dimension $\geq 2$ satisfying $S_{2}$ and $H_{*}^{1}\left(\mathcal{O}_{X}\right)=0$.

a) For any codimension two subscheme $V$ without embedded points, we associate to it the psi-equivalence class of coherent sheaves satisfying $T$ that contains the sheaf $\mathcal{I}_{V}$.

b) If furthermore $X$ satisfies $S_{3}$, then every psi-equivalence class of sheaves satisfying $T$ contains a sheaf of the form $\mathcal{I}_{V^{\prime}}(n)$, where $V^{\prime}$ is a codimension two subscheme without embedded points, for some $n \in \mathbb{Z}$.

c) Every psi-equivalence class of sheaves satisfying $T$ contains extraverti sheaves, unique up to stable equivalence. 
Proof. a) is (1.7)a.

b) is (1.8) together with (1.7)b.

c) is (1.11) plus (1.10).

Remark 1.13. If $X$ satisfies in addition condition $G_{2}$, Gorenstein in codimension 2 , then the condition $\mathcal{E} x t^{1}\left(\mathcal{E}, \mathcal{O}_{X}\right)=0$ implies by local duality depth $\mathcal{E} \geq 2$ at every point of codimension 2 , so that then $\mathcal{E}$ will satisfy condition $S_{2}$. In the presence of $G_{1}$, this makes $\mathcal{E}$ reflexive 3 , 1.9]. Thus, the extraverti sheaves of $(1.12) \mathrm{c}$ are reflexive, and we recover the construction of Nollet [12] and Nagel [1] by another route.

Furthermore, if $X$ is nonsingular, and we take $V$ to be locally Cohen-Macaulay of codimension 2 , then depth $\mathcal{I}_{V} \geq r-1$ in codimension $r$, and the same applies to $\mathcal{E}$. Then the condition $\mathcal{E} x t^{1}(\mathcal{E}, \mathcal{O})=0$ makes depth $\mathcal{E}=r$ in codimension $r$, i.e., $\mathcal{E}$ is a Cohen-Macaulay sheaf. Since $X$ is nonsingular, the extraverti sheaves associated to $V$ will be locally free. Then we recover the usual $N$-type resolution of $\mathcal{I}_{V}$.

\section{The Lazarsfeld-Rao property}

We preserve the hypotheses (1.1) and in addition we assume $X$ satisfies $S_{3}$. Let $H$ be the divisor class corresponding to $\mathcal{O}_{X}(1)$, i.e., whose fractional ideal is isomorphic to $\mathcal{O}_{X}(-1)$. If $Y \subseteq X$ is an effective divisor linearly equivalent to $n H$ for some $n$, then $Y$ is a Cartier divisor on $X$, and so the scheme $Y$ satisfies $S_{2}$. Thus we can speak of generalized divisors on $Y$ [4].

Definition 2.1. Let $V$ be a closed subscheme of pure codimension 2 of $X$, with no embedded components. We say another such subscheme $V^{\prime}$ is obtained by an elementary biliaison of height $h$ from $V$, if there exists an effective divisor $Y \sim n H$ on $X$ for some $n$, and a linear equivalence $V^{\prime} \sim V+h H$ on $Y$ for some $h \in \mathbb{Z}$. (Here by abuse of notation, $H$ denotes the divisor class of $\mathcal{O}_{X}(1)$ or $\mathcal{O}_{Y}(1)$ according to context.) The equivalence relation generated by elementary biliaisons will be called biliaison. (To be precise, we should call this notion

complete intersection biliaison or CI-biliaison, to distinguish it from the more general notion of Gorenstein biliaison that uses ACM divisors $Y$ on $X$ [4.)

Proposition 2.2. If $V_{1}$ and $V_{2}$ are closed subschemes of codimension 2 of $X$ as above that are equivalent for biliaison, then there is an integer $m \in \mathbb{Z}$ such that the ideal sheaves $\mathcal{I}_{V_{1}}$ and $\mathcal{I}_{V_{2}}(m)$ are equivalent for pseudo-isomorphism.

Proof. Let $V^{\prime} \sim V+h H$ as a divisor $Y \sim n H$ of $X$. Then $\mathcal{I}_{V^{\prime}, Y} \cong \mathcal{I}_{V, Y}(-h)$. On the other hand, $\mathcal{I}_{V^{\prime}, Y} \sim \mathcal{I}_{V^{\prime}, X}$ for psi and $\mathcal{I}_{V, Y} \sim \mathcal{I}_{V, X}$ for psi by (1.7). Hence $\mathcal{I}_{V^{\prime}} \sim \mathcal{I}_{V}(-h)$ for psi. Combining a sequence of elementary biliaisons gives the result. 
Remark 2.3. The converse of this proposition is the theorem of Rao: if the ideal sheaves $\mathcal{I}_{V_{1}}$ and $\mathcal{I}_{V_{2}}(n)$ are equivalent for psi, then $V_{1}$ and $V_{2}$ are equivalent for biliaison. The LazarsfeldRao property gives a more detailed structure of the biliaison equivalence class of codimension 2 subschemes. Both results are combined in the following theorem.

Theorem 2.4. Let $X$ be a projective scheme over an algebraically closed field $k$, equidimensional of dimension $N \geq 2$. Let $\mathcal{O}_{X}(1)$ be a very ample invertible sheaf. We assume that $X$ is integral, that it satisfies condition $S_{3}$ of Serre, and that $H_{*}^{1}\left(\mathcal{O}_{X}\right)=0$. We consider closed subschemes $V$ of codimension 2 equidimensional and without embedded components.

a) If $\mathcal{I}_{V_{1}}$ and $\mathcal{I}_{V_{2}}(n)$ are psi-equivalent for some $n \in \mathbb{Z}$, then $V_{1}$ and $V_{2}$ are equivalent for biliaison. Hence the biliaison equivalence classes of subschemes $V$ are in one-to-one correspondence with psi-equivalence classes (up to twist) of coherent sheaves satisfying condition $T$ (1.6).

b) If $V$ is a codimension 2 subscheme whose degree is not minimal in its biliaison equivalence class, then $V$ admits a strictly descending biliaison (i.e., there exists a divisor $Y \sim n H$ on $X$ containing $V$, and a subscheme $V^{\prime} \sim V+h H$ on $Y$ with $\left.h<0\right)$.

c) Any two subschemes $V, V^{\prime}$ in the same biliaison class, both of minimal degree, can be joined by a sequence of elementary biliaisons of height 0, i.e., linear equivalences on divisors $Y_{i} \sim n_{i} H$ on $X$.

The proof will follow after some preliminary results. The main idea is this: if $\mathcal{I}_{V_{1}}(a)$ and $\mathcal{I}_{V_{2}}(b)$ are equivalent for psi, then by (1.5) there exists a coherent sheaf $\mathcal{E}$ (satisfying $T$ by (1.8)) and elementary psi's from $\mathcal{E}$ to $\mathcal{I}_{V_{1}}(a)$ and $\mathcal{I}_{V_{2}}(b)$. Thus there are exact sequences

$$
\begin{aligned}
0 & \rightarrow \oplus_{i=1}^{r} \mathcal{O}\left(-a_{i}\right) \stackrel{\alpha}{\rightarrow} \mathcal{E} \rightarrow \mathcal{I}_{V_{1}}(a) \rightarrow 0 \\
0 & \rightarrow \oplus_{i=1}^{r} \mathcal{O}\left(-b_{i}\right) \stackrel{\beta}{\rightarrow} \mathcal{E} \rightarrow \mathcal{I}_{V_{2}}(b) \rightarrow 0
\end{aligned}
$$

where $\mathcal{E}$ has rank $r+1$. The maps $\alpha, \beta$ are defined by sections $s_{i} \in H^{0}\left(\mathcal{E}\left(a_{i}\right)\right)$ and $t_{i} \in$ $H^{0}\left(\mathcal{E}\left(b_{i}\right)\right)$. The proof proceeds by comparison of the integers $a_{i}, b_{i}$, and a careful study of the exact conditions for a section $s \in H^{0}(\mathcal{E}(n))$ to have a quotient $\mathcal{E}^{\prime}=\mathcal{E}(n) /(s)$ that satisfies $T$.

First we consider a special case.

Proposition 2.5. Let $X$ satisfy the hypotheses of (2.4), let $\mathcal{E}$ be a rank 2 coherent sheaf on $X$ satisfying $T$, and suppose there are codimension 2 subschemes $V_{1}, V_{2}$ and exact sequences

$$
0 \rightarrow \mathcal{O}\left(-a_{1}\right) \stackrel{\alpha}{\rightarrow} \mathcal{E} \rightarrow \mathcal{I}_{V_{1}}(a) \rightarrow 0
$$




$$
0 \rightarrow \mathcal{O}\left(-b_{1}\right) \stackrel{\beta}{\rightarrow} \mathcal{E} \rightarrow \mathcal{I}_{V_{2}}(b) \rightarrow 0 .
$$

Then $V_{2}$ is obtained from $V_{1}$ by an elementary biliaison of height $b-a$ on a suitable divisor $Y \sim n H$ on $X$.

Proof. Consider the composed map $\gamma: \mathcal{O}\left(-b_{1}\right) \stackrel{\beta}{\rightarrow} \mathcal{E} \rightarrow \mathcal{I}_{V_{1}}(a)$. If $\gamma$ is zero, then $\beta$ factors through $\mathcal{O}\left(-a_{1}\right)$. We get an injective map $\mathcal{O}\left(-b_{1}\right) \rightarrow \mathcal{O}\left(-a_{1}\right)$ whose cokernel is contained in $\mathcal{I}_{V_{2}}(b)$, hence is zero, because $\mathcal{I}_{V_{2}}(b)$ is torsion-free. Thus $a_{1}=b_{1}$, so $\alpha=\beta, a=b$, and $\mathcal{I}_{V_{1}}=\mathcal{I}_{V_{2}}$, so $V_{1}$ and $V_{2}$ are equal.

If $\gamma$ is not zero, then since $X$ is integral (and here is exactly where we use the hypothesis $X$ integral), it is injective. Let the cokernel be $\mathcal{F}$ :

$$
0 \rightarrow \mathcal{O}\left(-b_{1}\right) \stackrel{\gamma}{\rightarrow} \mathcal{I}_{V_{1}}(a) \rightarrow \mathcal{F} \rightarrow 0
$$

Let $Y$ be the divisor on $X$ defined by $\gamma$ followed by the inclusion of $\mathcal{I}_{V_{1}}(a)$ in $\mathcal{O}_{X}(a)$. Then $Y \sim\left(a+b_{1}\right) H$ on $X$, and $\mathcal{F} \cong \mathcal{I}_{V_{1}, Y}(a)$. A diagram chase shows that $\mathcal{F}$ also fits into another exact sequence

$$
0 \rightarrow \mathcal{O}\left(-a_{1}\right) \rightarrow \mathcal{I}_{V_{2}}(b) \rightarrow \mathcal{F} \rightarrow 0
$$

Hence $\mathcal{F} \cong \mathcal{I}_{V_{2}, Y}(b)$ also. Therefore $\mathcal{I}_{V_{1}, Y}(a) \cong \mathcal{I}_{V_{2}, Y}(b)$ and there is a linear equivalence $V_{2} \sim V_{1}+(b-a) H$ on $Y$. Thus $V_{2}$ is obtained by an elementary biliaison of height $b-a$ from $V_{1}$.

Proposition 2.6. Let $X$ be an integral projective scheme satisfying condition $S_{2}$ of Serre. Let $\mathcal{E}$ be a torsion-free coherent sheaf, locally free in codimension 1 . Let $W$ be a subvector space of $H^{0}(\mathcal{E})$, and let $\mathcal{E}_{0}$ be the subsheaf generated by $W$. Then the following conditions are equivalent:

(i) There exists a section $s \in W$ such that $\mathcal{E}^{\prime}=\mathcal{E} /(s)$ is torsion-free and locally free in codimension 1

(ii) (a) for all $x \in X$ of codimension 1 , $\operatorname{rank}\left(\mathcal{E}_{0} \otimes k(x) \stackrel{\sigma_{x}}{\rightarrow} \mathcal{E} \otimes k(x)\right) \geq 1$, and

(b) either rank $\mathcal{E}_{0} \geq 2$ or $\mathcal{E}_{0} \cong \mathcal{O}_{X}$ and $\mathcal{E} / \mathcal{E}_{0}$ is torsion-free and locally free in codimension 1 .

Proof (cf. [6, 1.4]) (i) $\Rightarrow$ (ii). Suppose given $s \in W$ such that $\mathcal{E}^{\prime}=\mathcal{E} /(s)$ is torsion-free and locally free in codimension 1 . Then there is a diagram

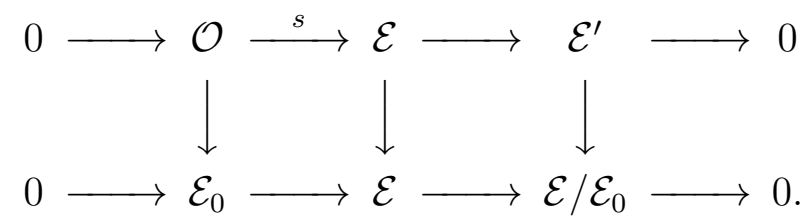


Let $x$ be a point of codimension 1 , and tensor with $k(x)$. Since $\mathcal{E}^{\prime}$ is locally free at $x$, we have

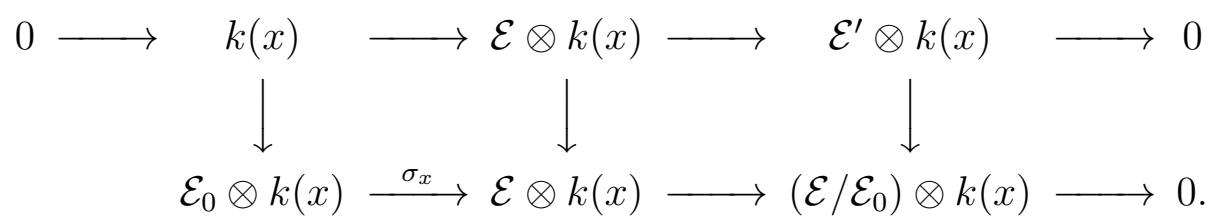

Thus it is clear that rank $\sigma_{x} \geq 1$. If rank $\mathcal{E}_{0} \geq 2$, there is nothing more to prove. If rank $\mathcal{E}_{0}=1$, then $\mathcal{E}_{0} / \mathcal{O} \subseteq \mathcal{E}^{\prime}$, and since $\mathcal{E}^{\prime}$ is torsion-free, we must have $\mathcal{E}_{0} \cong \mathcal{O}$ and $\mathcal{E} / \mathcal{E}_{0} \cong \mathcal{E}^{\prime}$ torsion-free and locally free in codimension 1 , as required.

(ii) $\Rightarrow$ (i). Assuming condition (ii), we will show that a general element $s \in W$ makes $\mathcal{E}^{\prime}=\mathcal{E} /(s)$ locally free in codimension 1 . Then the condition $\mathcal{E}$ torsion-free and $X$ satisfies $S_{2}$ will imply $\mathcal{E}^{\prime}$ also torsion-free.

Since $\mathcal{E}$ is locally free in codimension 1 , the condition that $\mathcal{E}^{\prime}$ be locally free at a point $x$ of codimension 1 is precisely that $s(x) \in \mathcal{E} \otimes k(x)$ be non-zero. Consider the bad locus $B=\{(s, x) \mid s(x)=0$ in $\mathcal{E} \otimes k(x)\}$ inside $W \times X$. From condition (ii) a) it is clear that $\mathcal{E}_{0} \neq 0$. If rank $\mathcal{E}_{0}=1$, then we know that $\mathcal{E}_{0} \cong \mathcal{O}_{X}$, and $\mathcal{E} / \mathcal{E}_{0}$ locally free in codimension 1 , so there is nothing to prove. So we may assume rank $\mathcal{E}_{0} \geq 2$.

Since $X$ is integral, there is a nonempty open set $U \subseteq X$ over which $\mathcal{E} / \mathcal{E}_{0}$ is locally free. For any $x \in U$, rank $\sigma_{x} \geq 2$ and so the fiber $B_{x}$ of $B$ over $x$ has codimension $\geq 2$ in $W$. Therefore $B_{U}=B \cap(W \times U)$ has dimension $\leq w-2+N$, where $w=\operatorname{dim} W$ and $N=\operatorname{dim} X$. Let $D=X-U$. At points $x \in D$ of codimension 1 in $X$, rank $\sigma_{x} \geq 1$. Hence rank $\sigma_{x} \geq 1$ over an open set $U^{\prime} \leq D$, and $\operatorname{dim} B_{U^{\prime}} \leq w-1+N-1$. Finally, there may be some subset $Z \subseteq D_{1}$ of codimension $\geq 2$ in $X$, for which $\operatorname{rank} \sigma_{x}=0$. Then $\operatorname{dim} B_{Z} \leq w+N-2$. All in all, we find $\operatorname{dim} B \leq w+N-2$. Looking at the projection of $B$ to $W$, we see that for a general $s \in W$, the fiber $B_{s} \subseteq X$ of $B$ over $s$ will have codimension $\geq 2$. Therefore $\mathcal{E}^{\prime}=\mathcal{E} /(s)$ will be locally free in codimension 1 , as required.

Remark 2.7. If $X$ satisfies the hypotheses (1.1) and also condition $S_{3}$ of Serre, and if the sheaf $\mathcal{E}$ of $(2.6)$ satisfies $T$, then the quotients $\mathcal{E}^{\prime}=\mathcal{E} /(s)$ of (2.6) will also satisfy $T$. Indeed the extra conditions 3$), 4)$ of (1.6) follow from the same conditions for $\mathcal{E}$ plus the condition $S_{3}$ on $X$.

Proposition 2.8. With the hypotheses of (2.4), suppose that $\mathcal{E}$ is a coherent sheaf satisfying $T$, and that there are codimension 2 subschemes $V$ and $W$ and exact sequences

$$
\begin{aligned}
0 & \rightarrow \oplus_{i=1}^{r} \mathcal{O}\left(-a_{i}\right) \stackrel{\alpha}{\rightarrow} \mathcal{E} \rightarrow \mathcal{I}_{V}(a) \rightarrow 0 \\
0 & \rightarrow \oplus_{i=1}^{r} \mathcal{O}\left(-b_{i}\right) \stackrel{\beta}{\rightarrow} \mathcal{E} \rightarrow \mathcal{I}_{W}(b) \rightarrow 0 .
\end{aligned}
$$

Assume that $a_{1} \leq a_{2} \leq \cdots \leq a_{r}$ and $b_{1} \leq b_{2} \leq \cdots \leq b_{r}$ and that $a_{i}=b_{i}$ for $i=1, \ldots, k-1$, and $a_{k}<b_{k}$ for some $k$. Then $W$ admits a strictly descending biliaison. 
Proof. Let $s_{i} \in H^{0}\left(\mathcal{E}\left(a_{i}\right)\right)$ and $t_{i} \in H^{0}\left(\mathcal{E}\left(b_{i}\right)\right)$ be the sections defining the maps $\alpha$ and $\beta$. The idea is to show that $t_{1}, \ldots, t_{k-1}, s_{k}, t_{k+1}, \ldots, t_{r}$ define another subscheme $W^{\prime}$ by an exact sequence

$$
0 \rightarrow \oplus_{i \neq k} \mathcal{O}\left(-b_{i}\right) \oplus \mathcal{O}\left(-a_{k}\right) \rightarrow \mathcal{E} \rightarrow \mathcal{I}_{W^{\prime}}\left(b^{\prime}\right) \rightarrow 0 .
$$

Then considering determinants of the locally free sheaves on an open subset of $X$ we find $b^{\prime}=b-b_{k}+a_{k}<b$. If we let $\mathcal{E}^{\prime}=\mathcal{E} /\left(t_{1}, \ldots, t_{k-1}, t_{k+1}, \ldots, t_{r}\right)$, then $\mathcal{E}^{\prime}$ is a rank 2 coherent sheaf with sections $t_{k}, s_{k}$ defining $W$ and $W^{\prime}$, respectively. According to (2.5), $W^{\prime}$ is then obtained from $W$ by an elementary biliaison of height $b^{\prime}-b<0$, and we get the desired result. This idea may not work with the original $s_{i}, t_{i}$, so we must modify them a little, without changing $V$ and $W$.

Step 1. We will show that for sufficiently general $s_{k}^{\prime} \in H^{0}\left(\mathcal{E}\left(a_{k}\right)\right)$, the quotient sheaf $\mathcal{E} /\left(t_{1}, \ldots, t_{k-1}, s_{k}^{\prime}\right)$ satisfies $T$ of rank $r+1-k$. To show this, consider the sheaf $\mathcal{F}=$ $\mathcal{E}\left(a_{k}\right) /\left(t_{1}, \ldots, t_{k-1}\right)$. This has $\mathcal{I}_{W}\left(b+a_{k}\right)$ as a quotient, hence satisfies $T$ by (1.8). We will apply (2.6) to $\mathcal{F}$ to show that it has a section $\bar{s}_{k}^{\prime}$ (which lifts to a section $s_{k}^{\prime} \in H^{0}\left(\mathcal{E}\left(a_{k}\right)\right)$ ) with quotient satisfying $T$.

Let $\mathcal{E}_{0}$ be the subsheaf of $\mathcal{E}\left(a_{k}\right)$ generated by global sections, and let $\mathcal{F}_{0}$ be the subsheaf of $\mathcal{F}$ generated by global sections. Then we have

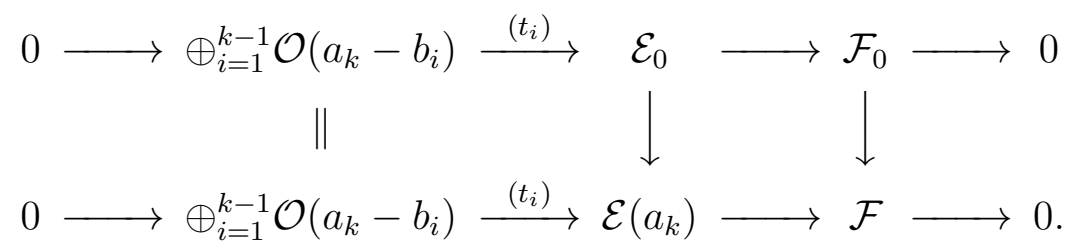

On the other hand, we know that $\mathcal{E} /\left(s_{1}, \ldots, s_{k}\right)$ satisfies $T$ and is of rank $r+1-k$, so for every point $x \in X$ of codimension 1 we must have rank $\left(\sigma_{x}\left(\mathcal{E}\left(a_{k}\right)\right): \mathcal{E}_{0} \otimes k(x) \rightarrow\right.$ $\left.\mathcal{E}\left(a_{k}\right) \otimes k(x)\right) \geq k$. Tensoring the diagram above with $k(x)$, it follows that $\operatorname{rank}\left(\sigma_{x}(\mathcal{F})\right.$ : $\left.\mathcal{F}_{0} \otimes k(x) \rightarrow \mathcal{F} \otimes k(x)\right) \geq 1$. Furthermore, if rank $\mathcal{F}_{0}=1$, then rank $\mathcal{E}_{0}=k$, so $\mathcal{E}_{0}$ must be equal to the subsheaf generated by $s_{1}, \ldots, s_{k}$, which is $\oplus_{i=1}^{k} \mathcal{O}\left(a_{k}-a_{i}\right)$. In this case $\mathcal{E}_{0} /\left(t_{1}, \ldots, t_{k-1}\right) \cong \mathcal{E}_{0} /\left(s_{1}, \ldots, s_{k-1}\right) \cong \mathcal{O}_{X}$ and $\mathcal{F} / \mathcal{F}_{0}=\mathcal{E}\left(a_{k}\right) / \mathcal{E}_{0}$ satisfies $T$.

So the conditions of (2.6) are satisfied (taking the whole space $H^{0}\left(\mathcal{E}\left(a_{k}\right)\right)$ ), and there is a section $s_{k}^{\prime} \in \mathcal{E}\left(a_{k}\right)$ so that $\mathcal{E}\left(a_{k}\right) /\left(t_{1}, \ldots, t_{k-1}, s_{k}^{\prime}\right)=\mathcal{F} /\left(\bar{s}_{k}^{\prime}\right)$ satisfies $T$. Note also that the image of $s_{k}^{\prime}$ in $\mathcal{I}_{W}\left(b+a_{k}\right)$ is nonzero. For if it were zero, then $s_{k}^{\prime} \in H^{0}\left(\oplus_{i=1}^{r}\left(a_{k}-b_{i}\right)\right)$. Since $a_{k}<b_{k}$, in fact $s_{k}^{\prime} \in H^{0}\left(\oplus_{i=1}^{k-1} \mathcal{O}\left(a_{k}-b_{i}\right)\right)$, which is impossible since $\bar{s}_{k}^{\prime} \neq 0$ in $H^{0}(\mathcal{F})$.

Step 2 (cf. proof of [1, 2.1]). Now we will show that for suitable choice of $t_{i}^{\prime}=t_{i}+$ $f_{i} t_{k}, i=k+1, \ldots, r$, where the $f_{i}$ are elements of $H^{0}\left(\mathcal{O}_{X}\left(b_{i}-b_{k}\right)\right)$, the sheaf $\mathcal{E}^{\prime \prime}=$ $\mathcal{E} /\left(t_{1}, \ldots, t_{k-1}, s_{k}^{\prime}, t_{k-1}^{\prime}, \ldots, t_{r}^{\prime}\right)$ will satisfy $T$ of rank 1 , hence be of the form $\mathcal{I}_{W^{\prime}}\left(b^{\prime}\right)$ for some codimension 2 scheme $W^{\prime}$. 
To do this, consider the sheaf $\mathcal{G}=\mathcal{E} /\left(t_{1}, \ldots, t_{r}, s_{k}^{\prime}\right)=\mathcal{E}^{\prime \prime} /\left(t_{k}\right)$. Since $\mathcal{G}$ is the quotient of $\mathcal{I}_{W}(b)$ by the image of $s_{k}^{\prime}$, it is a torsion sheaf supported along a divisor $Y$. Thus $\mathcal{E}^{\prime \prime}$ is locally free in codimension 1 except possibly along $Y$. For each generic point $y$ of an irreducible component of $Y$, we have $t_{1}(y), \ldots, t_{k-1}(y), s_{k}^{\prime}(y)$ linearly independent in $\mathcal{E} \otimes k(y)$, and similarly for $t_{1}(y), \ldots, t_{r}(y)$. Hence for general forms $f_{i} \in H^{0}\left(\mathcal{O}_{X}\left(b_{i}-b_{k}\right)\right)$, letting $t_{i}^{\prime}=t_{i}+f_{i} t_{k}, i=k+1, \ldots, r$, we will have $t_{1}(y), \ldots, t_{k-1}(y), s_{k}^{\prime}(y), t_{k+1}^{\prime}(y), \ldots, t_{r}^{\prime}(y)$ linearly independent, and so $\mathcal{E}^{\prime \prime}$ will be locally free at $y$. We can do this simultaneously for the finite number of generic points $y$ of $Y$. Thus $\mathcal{E}^{\prime \prime}$ will be locally free of rank 1 in codimension 1 , hence will satisfy $T$ and be of the form $\mathcal{I}_{W^{\prime}}\left(b^{\prime}\right)$.

Step 3. Now take $\mathcal{E}^{\prime}=\mathcal{E} /\left(t_{1}, \ldots, t_{k-1}, t_{k+1}^{\prime}, \ldots, t_{r}^{\prime}\right)$. Then $\mathcal{E}^{\prime}$ satisfies $T$ of rank 2 , and $\mathcal{E}^{\prime} /\left(t_{k}\right)=\mathcal{I}_{W}(b)$, and $\mathcal{E}^{\prime} /\left(s_{k}^{\prime}\right)=\mathcal{I}_{W^{\prime}}\left(b^{\prime}\right)$. Then, as explained above, using $(2.5), W^{\prime}$ is obtained by a strictly descending elementary biliaison from $W$.

Proposition 2.9. Suppose given $V, W$ and exact sequences as in (2.8) with $a_{i}=b_{i}$ for each $i$. Then $W$ is obtained from $V$ by a finite number of elementary biliaisons of height zero.

Proof. By induction on rank $\mathcal{E}=r+1$. Note first that by computing determinants, since $a_{i}=b_{i}$ for all $i$, we find $a=b$. If $\operatorname{rank} \mathcal{E}=2$, this is just (2.5). So suppose rank $\mathcal{E} \geq 3$, i.e., $r \geq 2$. As in the proof of (2.8), let $s_{i}$ and $t_{i}$ define the maps $\alpha$ and $\beta$.

Applying (2.6) to the sheaf $\mathcal{F}=\mathcal{E} /\left(s_{1}, \ldots, s_{r-1}\right)$, since $\mathcal{E}\left(a_{r}\right)$ has one section $s_{r}$ making a quotient satisfying $T$, it follows that if we take a general section $s^{\prime} \in H^{0}\left(\mathcal{E}\left(a_{r}\right)\right)$, then $\mathcal{E}^{\prime}=\mathcal{E} /\left(s_{1}, \ldots, s_{r-1}, s^{\prime}\right)$ will be equal to $\mathcal{I}_{V^{\prime}}(a)$ for some codimension 2 subscheme $V^{\prime}$. Then using (2.5) applied to $\mathcal{F}$, we find $V$ and $V^{\prime}$ are related by an elementary biliaison of height zero.

Doing the same with $\mathcal{G}=\mathcal{E} /\left(t_{1}, \ldots, t_{r-1}\right)$, we find that for sufficiently general $s^{\prime} \in$ $H^{0}\left(\mathcal{E}\left(a_{r}\right)\right)$, which we can take to be the same as the $s^{\prime}$ above $(!)$, the quotient $\mathcal{E} /\left(t_{1}, \ldots, t_{r-1}, s^{\prime}\right)$ will define a subscheme $W^{\prime}$, related by one elementary biliaison of height zero to $W$.

Now $V^{\prime}$ and $W^{\prime}$ both have resolutions of the above form using the sheaf $\mathcal{E}^{\prime}=\mathcal{E} /\left(s^{\prime}\right)$, of rank one less, so by the induction hypothesis, $V^{\prime}$ and $W^{\prime}$ are related by a finite sequence of elementary biliaisons of height zero, and we are done.

Proof of (2.4). Suppose given subschemes $V$ and $W$ such that $\mathcal{I}_{V}(a)$ and $\mathcal{I}_{W}(b)$ are psiequivalent. Then by (1.5) there is a coherent sheaf $\mathcal{E}$ satisfying $T$ and exact sequences as in the statement of (2.8). If $a_{i}$ is not equal to $b_{i}$ for all $i$, then by (2.8), one of the two subschemes admits a descending biliaison to a subscheme of lower degree. Since the degree of a subscheme is always nonnegative, we can proceed inductively, and after a finite number of steps we arrive at a situation where $a_{i}=b_{i}$ for all $i$. Then (2.9) applies to show they are equivalent by a finite number of biliaisons of height zero. 
This proves a) of the theorem. For b), given $V$ not of minimal degree, take any $W$ of minimal degree in the same biliaison class. Then in (2.8) it must be $V$ that admits the descending biliaison.

As for c), if $V$ and $W$ are both of minimal degree, neither one can admit a descending biliaison, so the $a_{i}$ must be equal to the $b_{i}$, and (2.9) applies.

Remark 2.10. There is one special case of the theorem that merits special attention. It might happen in the course of the proof that one of the subschemes obtained by a descending biliaison is empty. The theorem and its proof still hold, provided that we allow the empty scheme. The psi class of the corresponding coherent sheaves is the class containing the dissocié sheaves. This corresponds to one biliaison equivalence class, the one containing complete intersections of hypersurfaces $Y \sim n H$ and $Y^{\prime} \sim n^{\prime} H$ in $X$. The schemes of minimal degree in this class are the empty scheme.

This may be regarded as unsatisfactory, so we prove separately that for the biliaison class corresponding to the dissocie sheaves, the results of the theorem hold also if we restrict our attention only to the nonempty schemes.

We have only to consider the case where in the proof of (2.8) we might obtain an empty scheme $W^{\prime}$. In that case, $W \sim m H$ on a hypersurface $Y \sim n H$ in $X$. Since $H^{1}\left(\mathcal{O}_{X}(m-n)\right)=$ 0 , it follows that $H^{0}\left(\mathcal{O}_{X}(m)\right) \rightarrow H^{0}\left(\mathcal{O}_{Y}(m)\right)$ is surjective, so $W$ is a complete intersection of $Y$ with $Y^{\prime} \sim m H$. If $m \geq 2$, we can make a descending biliaison to $W^{\prime} \sim H$ on $Y$. If $m=1$ and $n \geq 2$, we regard $W$ as a divisor on $Y^{\prime} \sim H$ on $X$ and again make a descending biliaison to $W^{\prime} \sim H$ on $Y^{\prime}$. If $m=n=1$, I claim $W$ is of minimal degree among the nonempty schemes in this biliaison class. For if $V$ was something of lower degree, according to the theorem there would be a sequence $V=V_{1}, V_{2}, \ldots, V_{r}$ of descending biliaisons, with $V_{r}$ the empty scheme. In that case, as we have seen, $V_{r-1}$ would be a complete intersection of degree $\geq W$, which is impossible. The same argument shows that any nonempty scheme of minimal degree is of the same form as $W$. Now $W$ has a resolution of the form $0 \rightarrow$ $\mathcal{O}(-2) \rightarrow \mathcal{O}(-1) \oplus \mathcal{O}(-1) \rightarrow \mathcal{I}_{W} \rightarrow 0$. Given two of these, they have the same $\mathcal{E}$ and the same $a_{i}$, so by $(2.9)$ they are joined by a sequence of biliaisons of height zero.

Remark 2.11. In case $X=\mathbb{P}^{n}$, the codimension two schemes in the biliaison class of the empty scheme are the ACM schemes. So we have just shown (2.10) that any ACM codimension 2 scheme admits descending elementary biliaisons to a minimal one, which is just a linear variety $\mathbb{P}^{n-2}$ in $\mathbb{P}^{n}$.

Remark 2.12. A consequence of the theorem is that a variety $V$ that is not of minimal degree can be reached by a sequence of strictly ascending elementary biliaisons from one of minimal degree. For curves in $\mathbb{P}^{3}$, this is Strano's theorem [15], answering a question of [9, p. 93], showing that the deformation in earlier proofs of the Lazarsfeld-Rao property is not necessary. 
Remark 2.13. If we wish to prove only (2.4a), namely that psi equivalence of ideal sheaves implies biliaison equivalence of subschemes, we can remove the hypothesis that $X$ is integral. We prove this by the original method of Rao. First of all, in (2.5) instead of comparing $\alpha$ to $\beta$, we compare them both to $\gamma: \mathcal{O}(-c) \rightarrow \mathcal{E}$, where $c$ is chosen sufficiently large that $\mathcal{E}(c)$ is generated by global sections. Then the induced maps $\mathcal{O}(-c) \rightarrow \mathcal{I}_{V_{1}}(a)$ and $\mathcal{O}(-c) \rightarrow \mathcal{I}_{V_{2}}(b)$ will both be injective, and we find that $V_{1}$ and $V_{2}$ are now related by two biliaisons of large heights.

For sheaves of arbitrary rank, we follow the plan of proof of (2.9), but take the section $s^{\prime}$ of that proof to be a general section of $\mathcal{E}(n)$ for $n \gg 0$. Then we can make $s^{\prime}$ independent of the other sections and proceed as in that proof, except that the biliaisons are now up and down of large heights.

Corollary 2.14. a) With the hypotheses of (2.4), the biliaison equivalence classes of codimension two subschemes are in one-to-one correspondence with stable equivalence classes of extraverti sheaves satisfying $T$ (up to twist).

b) If furthermore $X$ also satisfies $G_{2}$, then the biliaison classes of codimension two subschemes are in one-to-one correspondence with stable equivalence classes of reflexive extraverti sheaves satisfying $T$ (up to twist).

Proof. This is simply a restatement of (2.4), using (1.12) and (1.13). Part b) is the theorem of Nollet [12, 2.12] in the case $X=\mathbb{P}^{n}$, and of Nagel [11, 6.4] in the case $X$ is an integral arithmetically Gorenstein scheme. Nagel also proved [11, 7.3] the weaker form of the Lazarsfeld-Rao property (2.4b) allowing a deformation before making a descending biliaison.

\section{Cohomological characterization of biliaison classes}

For curves in $\mathbb{P}^{3}$, the original theorem of Rao [13] says that two curves $C_{1}, C_{2}$ are in the same biliaison equivalence class if and only if their Rao modules $M_{1}, M_{2}$ are isomorphic up to twist. The Rao module of a curve $C$ is $M=H_{*}^{1}\left(\mathcal{I}_{C}\right)$. It was proved by first relating biliaison classes of curves to stable equivalence classes of locally free sheaves, and then using a theorem of Horrocks [7. The theorem of Horrocks is difficult to generalize to higher dimensions, but in this section we give a version for an arithmetically Gorenstein scheme $X$ of dimension 3 .

Proposition 3.1. Let $X$ be a 3-dimensional projective ACM scheme, and let $S=H_{*}^{0}\left(\mathcal{O}_{X}\right)$.

a) Each psi equivalence class of coherent sheaves $\mathcal{E}$ satisfying $T$ determines a finite length graded $S$-module $M=H_{*}^{1}(\mathcal{E})$.

b) Conversely, for each finite length graded $S$-module $M$, there is a locally free extraverti coherent sheaf $\mathcal{E}$ satisfying $T$, such that $H_{*}^{1}(\mathcal{E}) \cong M$. 
Proof. For part a) we first show that if $0 \rightarrow \mathcal{L} \rightarrow \mathcal{E} \rightarrow \mathcal{E}^{\prime} \rightarrow 0$ is an elementary psi, then $H_{*}^{1}(\mathcal{E}) \cong H_{*}^{1}\left(\mathcal{E}^{\prime}\right)$. This is because $X$ being ACM implies $H_{*}^{i}\left(\mathcal{O}_{X}\right)=0$ for $i=1$, 2. Serre's theorem tells us that $H^{1}(\mathcal{E}(n))=0$ for $n \gg 0$. To show that $M$ is of finite length, we must show also $H^{1}(\mathcal{E}(-n))=0$ for $n \gg 0$. Let $\omega$ be the dualizing sheaf on $X$. Then $H^{1}(\mathcal{E}(m))$ is dual to $\operatorname{Ext}_{X}^{2}(\mathcal{E}, \omega(n))$. For $n \gg 0$, the sheaves $\mathcal{E} x t^{i}(\mathcal{E}, \omega(n))$ have no higher cohomology, so the spectral sequence of local and global Ext gives $\operatorname{Ext}^{2}(\mathcal{E}, \omega(n))=H^{0}\left(\mathcal{E} x t^{2}(\mathcal{E}, \omega(n))\right)$. Now we use local duality at a closed point $x \in X$. There $\mathcal{E} x t^{2}(\mathcal{E}, \omega)_{x}$ is dual to $H_{x}^{1}(\mathcal{E})$, and this is zero because depth $\mathcal{E}_{x} \geq 2$ by condition $T$.

For part b), let $M$ be a finite length graded $S$-module. Take a resolution

$$
0 \rightarrow E \rightarrow L_{1} \rightarrow L_{0} \rightarrow M \rightarrow 0
$$

of graded $S$-modules, with $L_{i}$ free, and $E$ the second syzygy. Passing to associated sheaves we get

$$
0 \rightarrow \mathcal{E} \rightarrow \mathcal{L}_{1} \rightarrow \mathcal{L}_{0} \rightarrow 0
$$

This shows that $\mathcal{E}$ is locally free and $H_{*}^{1}(\mathcal{E}) \cong M$. Also $\operatorname{det} \mathcal{E}=\left(\operatorname{det} \mathcal{L}_{1}\right)\left(\operatorname{det} \mathcal{L}_{0}\right)^{-1}$, so $\mathcal{E}$ satisfies $T$. Taking duals, we find

$$
0 \rightarrow \mathcal{L}_{0}^{\vee} \rightarrow \mathcal{L}_{1}^{\vee} \rightarrow \mathcal{E}^{\vee} \rightarrow 0
$$

and $\mathcal{E} x t^{1}(\mathcal{E}, \mathcal{O})=0$. From this, again using $X$ is $\mathrm{ACM}$, we find $H_{*}^{1}\left(\mathcal{E}^{\vee}\right)=0$, so $\mathcal{E}$ is extraverti.

Theorem 3.2. Now assume that $X$ is a 3-dimensional arithmetically Gorenstein scheme, i.e., $X$ is $\mathrm{ACM}$ and $S=H_{*}^{0}\left(\mathcal{O}_{X}\right)$ is a Gorenstein ring.

a) To each extraverti coherent sheaf $\mathcal{E}$ satisfying $T$ we will associate a maximal CohenMacaulay module $P$ over the ring $S$, defined up to stable equivalence, and a map $\alpha: P^{\vee} \rightarrow$ $M^{*} \rightarrow 0$, where $M^{*}$ is the dual of the finite length module $M=H_{*}^{1}(\mathcal{E})$.

b) If $\mathcal{E}_{1}$ and $\mathcal{E}_{2}$ are two such sheaves with the same associated modules $M_{1} \cong M_{2}$, and if the associated maximal Cohen-Macaulay modules $P_{1}, P_{2}$ are stably equivalent and the maps $\alpha_{1}: P_{1}^{\vee} \rightarrow M^{*}$ and $\alpha_{2}: P_{2}^{\vee} \rightarrow M^{*}$ are compatible with the stable equivalence, then $\mathcal{E}_{1}$ and $\mathcal{E}_{2}$ are stably equivalent.

c) Given a finite-length graded S-module $M$, a graded maximal Cohen-Macaulay module $P$ whose associated sheaf $\mathcal{P}$ is orientable, and given a map $\alpha: P^{\vee} \rightarrow M^{*} \rightarrow 0$, there exists an extraverti sheaf $\mathcal{E}$ satisfying $T$ that gives rise to this triple, as above.

Proof. a) Given $\mathcal{E}$ extraverti satisfying $T$, let $E=H_{*}^{0}(\mathcal{E})$, and take a resolution of graded $S$-modules

$$
0 \rightarrow P \rightarrow L_{1} \rightarrow L_{0} \rightarrow E \rightarrow 0
$$


where $L_{0}, L_{1}$ are free and $P$ is the kernel. Since $E$ has depth $\geq 2$ at the irrelevant prime $\mathfrak{m}$ of $S$, we see that $P$ has depth 4 , so $P$ is a maximal Cohen-Macaulay module. Taking duals we obtain an exact sequence

$$
0 \rightarrow E^{\vee} \rightarrow L_{0}^{\vee} \rightarrow L_{1}^{\vee} \rightarrow P^{\vee} \stackrel{\alpha}{\rightarrow} M^{*} \rightarrow 0 .
$$

To see this, first consider $\operatorname{Ext}_{S}^{1}(E, S)$. This is dual on $S$ to $H_{\mathfrak{m}}^{3}(E)$, which is isomorphic to $H_{*}^{2}(\mathcal{E})$ on $X$. This in turn is dual to $\operatorname{Ext}_{X, *}^{1}(\mathcal{E}, \mathcal{O})$ which is zero because $\mathcal{E}$ is extraverti, using the exact sequence of low degree terms of the spectral sequence mentioned in the proof of (1.10). Hence $\operatorname{Ext}_{S}^{1}(E, S)=0$. Secondly, $\operatorname{Ext}_{S}^{2}(E, S)$ is dual to $H_{\mathfrak{m}}^{2}(E)$, which is isomorphic to $H_{*}^{1}(\mathcal{E})$, which is $M$.

In this way we obtain the maximal $\mathrm{CM}$ module $P$, determined up to stable equivalence on $S$, and the map $\alpha: P^{\vee} \rightarrow M^{*} \rightarrow 0$.

b) Now suppose $\mathcal{E}_{1}$ and $\mathcal{E}_{2}$ are two such sheaves, with isomorphic associated modules $M_{1} \cong M_{2}=M$ and stably equivalent maximal $\mathrm{CM}$ modules $P_{1}$ and $P_{2}$, and compatible maps $\alpha_{1}, \alpha_{2}$. Then $E_{1}^{\vee}$ and $E_{2}^{\vee}$ both occur in resolutions of $\alpha: P^{\vee} \rightarrow M^{*} \rightarrow 0$ as above, and hence $E_{1}^{\vee}$ and $E_{2}^{\vee}$ are stably equivalent graded $S$-modules. It follows that $\mathcal{E}_{1}$ and $\mathcal{E}_{2}$ are stably equivalent sheaves.

c) Given $\alpha: P^{\vee} \rightarrow M^{*} \rightarrow 0$, take a resolution

$$
0 \rightarrow E^{\prime} \rightarrow L_{1}^{\prime} \rightarrow L_{0}^{\prime} \rightarrow P^{\vee} \rightarrow M^{*} \rightarrow 0
$$

Then let $E=E^{\prime \vee}$ and let $\mathcal{E}$ be the associated sheaf. One checks easily that $\mathcal{E}$ is extraverti satisfying $T$ and gives rise to $M, P, \alpha$ as required.

Corollary 3.3. Let $X$ be an arithmetically Gorenstein scheme of dimension 3. Then to each curve $C \subseteq X$ is associated a maximal Cohen-Macaulay module $P$ over the ring $S=H_{*}^{0}\left(\mathcal{O}_{X}\right)$, and a map $\alpha: P^{\vee} \rightarrow M^{*} \rightarrow 0$, where $M=H_{*}^{1}\left(\mathcal{I}_{C}\right)$ is the Rao module of $C$. Two curves $C_{1}$ and $C_{2}$ are in the same biliaison equivalence class if and only if (up to shift) $M_{1} \cong M_{2}$, $P_{1}, P_{2}$ are stably equivalent, and $\alpha_{1}, \alpha_{2}$ are compatible with this isomorphism on a stable equivalence. Furthermore, every triple $(M, P, \alpha)$ as in $(3.2 \mathrm{c})$ occurs for some biliaison class of curves.

Proof. Given $C$, take any extraverti sheaf $\mathcal{E}$ in the psi equivalence class of $\mathcal{I}_{C}$ (1.12). Then we obtain the associated $P$ and $\alpha$ by (3.2). Now if two curves $C_{1}$ and $C_{2}$ have compatible $M_{i}, P_{i}$, and $\alpha_{i}$, up to shift, the corresponding sheaves $\mathcal{E}_{1}$ and $\mathcal{E}_{2}$ are stably equivalent, up to shift, by the theorem. This in turn implies $C_{1}$ and $C_{2}$ are in the same biliaison equivalence class by (2.4), cf. also (2.14).

Remark 3.4. In the special case $X=\mathbb{P}^{3}$, the maximal Cohen-Macaulay module is free, since $S$ is a regular ring. Thus the condition of (3.3) boils down to $M_{1} \cong M_{2}$, which is 
the original theorem of Rao. Note that the statement "every maximal Cohen-Macaulay module is free" over a local Cohen-Macaulay ring implies that every module has a finite free resolution, and so the ring is regular. Thus $\mathbb{P}^{3}$ is the only arithmetically Gorenstein scheme for which the isomorphism of Rao modules is sufficient to imply biliaison equivalence of curves.

For a two-dimensional arithmetically Gorenstein scheme, there is a much simpler analogous result.

Proposition 3.5. Let $X$ be an arithmetically Gorenstein scheme of dimension 2. There is a natural one-to-one correspondence between biliaison classes of 0-dimensional closed subschemes $Z$ and stable equivalence classes of maximal Cohen-Macaulay modules over the ring $S=H_{*}^{0}\left(\mathcal{O}_{X}\right)$ whose associated sheaf $\mathcal{E}$ is orientable.

Proof. To any 0-dimensional subscheme $Z$ we associate an extraverti sheaf $\mathcal{E}$ in the psiequivalence class of $\mathcal{I}_{Z}$ (1.11). Then $\mathcal{E}$ is reflexive (1.13) and hence locally Cohen-Macaulay on $X$. Since $X$ is arithmetically Gorenstein, the condition $H_{*}^{1}\left(\mathcal{E}^{\vee}\right)=0$ implies by Serre duality that $H_{*}^{1}(\mathcal{E})=0$. Hence $E=H_{*}^{0}(\mathcal{E})$ is a maximal Cohen-Macaulay module over $S$. Now (2.14) shows that biliaison equivalence classes of $Z$ correspond to stable equivalence classes of $\mathcal{E}$ and hence of $E$. The condition orientable just requires that $\mathcal{E}$ be locally free in codimension 1 and its determinant be isomorphic to $\mathcal{O}_{X}(\ell)$ for some $\ell$ on $X$ minus a finite number of points.

\section{References}

[1] Ballico, E., Bolondi, G., and Migliore, J. C., The Lazarsfeld-Rao problem for liaison classes of two-codimensional subschemes of $\mathbb{P}^{n}$, Amer. J. Math. 113 (1991) 117-128.

[2] Bolondi, G., and Migliore, J. C., The Lazarsfeld-Rao property on an arithmetically Gorenstein variety, Manusc. Math. 78 (1993) 347-368.

[3] Hartshorne, R., Generalized divisors on Gorenstein schemes, K-theory 8 (1994) 287-339.

[4] Hartshorne, R., Generalized divisors and biliaison, preprint.

[5] Hartshorne, R., Martin-Deschamps, M., and Perrin, D., Un théorème de Rao pour les familles de courbes gauches, J. Pure Appl. Algebra 155 (2001) 53-76.

[6] Hartshorne, R., Martin-Deschamps, M., and Perrin, D., Construction de familles minimales de courbes gauches, Pacific J. Math. 194 (2000) 97-116. 
[7] Horrocks, G., Vector bundles on the punctured spectrum of a local ring, Proc. Lond. Math. Soc. 14 (1964) 689-713.

[8] Lazarsfeld, R., and Rao, P., Linkage of general curves of large degree, Springer LNM 997 (1983) 267-289.

[9] Martin-Deschamps, M., and Perrin, D., Sur la classification des courbes gauches, Astérisque 184-185 (1990).

[10] Migliore, J. C., Introduction to Liaison Theory and Deficiency Modules, Birkhäuser, Boston (1998).

[11] Nagel, U., Even liaison classes generated by Gorenstein linkage, J. Algebra 209 (1998) $543-584$.

[12] Nollet, S., Even linkage classes, Trans. AMS 348 (1996) 1137-1162.

[13] Rao, P., Liaison among curves in $\mathbb{P}^{3}$, Invent. Math. 50 (1979) 205-217.

[14] Rao, P., Liaison equivalence classes, Math. Ann. 258 (1981) 169-173.

[15] Strano, R., Biliaison classes of curves in $\mathbb{P}^{3}$. 